\title{
La extensión universitaria como herramienta de intervención en los conflictos socioambientales
}

\author{
María de los Ángeles Gutiérrez \\ mariadla_gutierrez@yahoo.com.ar
}

Florencia Yanniello

florenciayanniello@gmail.com

\section{Santiago Elisio}

santiago_elisio@hotmail.com

Darío Andrinolo

dandrinolo@yahoo.com
Programa Ambiental de Extensión Universitaria (PAEU) / Centro de Investigaciones del Medio Ambiente (CIMA) - Facultad de Ciencias Exactas, Universidad Nacional de La Plata, Argentina.
A 100 años de la Reforma Universitaria de 1918 /

Desafíos de gestión

RECEPCIÓN: 20/06/17

ACEPTACIÓN FINAL: 24/10/17

\section{Resumen}

La contaminación ambiental se ha instalado en las últimas décadas en distintos ámbitos; sin embargo, las conceptualizaciones, las formas de pensar esta problemática transversal y los matices son diversos según los modelos de desarrollo desde donde se la analice. La universidad pública no está exenta de estas tensiones, ya que coexisten en su interior distintas maneras de considerar los conflictos ambientales y de abordarlos desde sus tres pilares fundamentales (docencia, investigación y extensión).

En 2010, la Facultad de Ciencias Exactas de la Universidad Nacional de La Plata creó el Programa Ambiental de Extensión Universitaria como un espacio donde pensar a la Universidad frente a los conflictos ambientales de nuestra región y como un motor en el desarrollo de políticas a través del diálogo con los actores involucrados. Se trabaja en la construcción de posibles propuestas hacia una sociedad más igualitaria, un desarrollo económicamente sustentable, socialmente justo y un ambiente sano.

\section{Palabras clave}

- Conflictos ambientales

- Extensión universitaria

- Programa Ambiental de Extensión Universitaria

\section{Resumo}

A poluição ambiental foi instalada nas últimas décadas em varios campos; no entanto, os conceitos, as nuances e formas de pensar esta questão transversal, são diferentes, dependendo do modelo de desenvolvimento de onde é analisado. A universidade pública não é isenta destas tensões, por que dentro de ela coexistem diferentes formas de pensar os conflitos ambientais e de resolvêlos através do seus três pilares (ensino, pesquisa e extensão).

Em 2010, a faculdade de ciências exatas criou o Programa de Extensão Universitária, como um lugar onde se pensa a universidade frente diferentes conflitos ambientais de nossa região. assim como o motor no desenvolvimento de políticas através do diálogo com diferentes atores envolvidos. Ele trabalha na construção de possíveis propostas para uma sociedade mais igualitária socialmente justa, economicamente desenvolvimento sustentável e um ambiente saudável.

Palavras-chave

- Conflitos ambientais

- Extensão universitária

- Programa Ambiental de Extensión Universitaria
Para citación de este artículo

Gutiérrez, M.; Yanniello, F.; Elisio, S. y Andrionolo, D. (2017). La extensión universitaria como herramienta de intervención en los conflictos socioambientales. Revista + E versión en línea, 7(7), 204-213. Santa Fe, Argentina: Ediciones UNL. 


\section{Línea del tiempo: la extensión universitaria en perspectiva} La incorporación de la extensión en las universidades latinoamericanas en el siglo XIX ha representado un cambio de ideales educativos, científicos y humanísticos. Sin embargo, su práctica es anterior a su inclusión como una de las bases doctrinarias del Movimiento Reformista.

María Caldelari analiza la historia de la extensión universitaria en Argentina y la define, en una primera etapa llevada a cabo por los reformadores (Joaquín V. González, Rodolfo Rivarola), como una "extensión oligárquica":

"Las primeras formas de extensión, aquellas ligadas a la acción cultural, están entretejidas con una concepción de universidad donde una de sus funciones prioritarias es cultural... Y las actividades serán organizadas de manera vertical, manteniendo las jerarquías tanto en lo que se refiere a quienes están autorizados a hablar, como en cuanto a la elección de los conocimientos que serán puestos en circulación". (Caldelari, 2002:55)

El modelo de extensión reformista sufrió los avatares de los diferentes golpes de Estado, que repercutieron en los objetivos y alcances de las universidades públicas, redireccionándose en cada proceso político.

En la década del ' 90 , el compromiso de la universidad con la sociedad fue a través de las empresas y la ejecución de políticas sociales descentralizadas. Según Argumedo: "El proyecto reformista de formar profesionales comprometidos, es reemplazado por el de producir profesionales altamente calificados para consumo empresarial" (2002:297).

La reforma del estatuto de la Universidad Nacional de La Plata (UNLP), en su Título VIII, artículo $106^{\circ}$ de la extensión universitaria, plantea que: "La Universidad considera la Extensión Universitaria como uno de los medios de realizar su función social" (UNLP, 1996:25). En ese marco se creó específicamente un departamento dedicado a este pilar.

Por otro lado, el Consejo Interuniversitario Nacional (CIN) en su Acuerdo Plenario $N^{\circ}$ 262/97, sobre la inclusión de la función extensión en el presupuesto 1998 para el sistema universitario, define a la extensión como:

"un proceso de comunicación entre la Universidad y la Sociedad, basado en el conocimiento científico, tecnológico, cultural, artístico, humanístico, acumulado en la institución y en su capacidad de formación educativa, con plena conciencia de su función social". (CIN; 1997)

En la actualidad, la reforma estatutaria de la UNLP plantea otro concepto de extensión:

"cuyos propósitos deben contribuir a la solución de las más diversas problemáticas sociales, la toma de decisiones y la formación de opinión, con el objeto de generar conocimiento a través de un proceso de integración con el medio y contribuir al desarrollo social". (2008:5)

Esta visión representa la expresión de un desarrollo integral de la extensión universitaria, superador de la extensión altruista o meramente divulgadora. Esta mirada incorpora el concepto de democratización del saber, asume la función social de contribuir a mejorar la calidad de vida de la sociedad y apunta a la transformación social y económica de los pueblos (González y González, 2003).

La visión integral extensionista pone a cada persona en un espacio sinérgico para la consecución de objetivos comunes de desarrollo social, cultural, ambiental y político, donde la universidad no se despoja de sus capacidades sino que las pone al servicio de una causa común.

En este marco, en la UNLP se vienen implementando diferentes herramientas que fortalecen la articulación entre la Universidad, las organizaciones sociales y productivas y los distintos niveles de gobierno (municipal, provincial y nacional). Un ejemplo de esto es el Consejo Social, creado en 2010, un órgano multisectorial dependiente del rectorado de la UNLP que permite planificar acciones para el desarrollo productivo y la recuperación de los derechos esenciales del conjunto de la población. En este sentido, los distintos programas y proyectos de extensión de las facultades representan el vehículo para generar respuestas a las demandas existentes, dentro de los cuales se encuentra el Programa Ambiental de Extensión Universitaria (PAEU).

\section{Qué entendemos por extensión desde el PAEU}

Este programa se creó en el año 2010 como un espacio desde donde pensar y movilizar a la Universidad frente a los distintos conflictos socioambientales de nuestra región y como motor en el desarrollo de políticas que inserten a aquella en estas problemáticas.

La construcción de posibles soluciones y propuestas resulta necesariamente de un proceso de diálogo con los diferentes actores involucrados, desde vínculos igualitarios, incorporando al ambiente como sujeto de derecho y a la sociedad como voz y parte del mismo.

Desde un enfoque institucional, la generación de programas de extensión universitaria ha sido un punto importante en la consolidación de la historia extensionista de la Facultad de Ciencias Exactas de la UNLP. Los programas nuclean proyectos de extensión que comparten afinidad temática, promueven una fuerte interacción entre sus integrantes y mejoran la gestión de los recursos con el fin de elaborar respuestas coordinadas y potenciadas a la comunidad. A su vez, los programas se establecen como lugares y puntos de referencia, de manera de ampliar los espacios universitarios preexistentes desde donde coordinar con la comunidad y otras instituciones. 
La dedicación extensionista nos pone continuamente en tensión y reflexión respecto de la función social y ambiental de la universidad pública. Los programas cumplen un rol fundamental hacia dentro de la universidad como expresión legítima de las nuevas formas de producción teórica y práctica de conocimiento, que se centran en la resolución de problemáticas concretas. Este proceso impacta naturalmente en la forma de pensar y hacer docencia y ciencia en la universidad. EI PAEU promueve una mirada multidisciplinaria a través de profesionales y estudiantes de distintas carreras de las ciencias exactas, sociales y naturales e ingeniería, en conjunto con los actores sociales e impulsando su participación.

En nuestra experiencia particular como programa ambiental, hemos abordado diferentes problemáticas de la región con el objetivo de participar en el esfuerzo común por alcanzar la soberanía ambiental, es decir, la potestad de los pueblos de decidir cómo habitar sus territorios.

Desde este marco de acción, buscamos una visión más holística del conocimiento, que nos ponga en un plano horizontal con otros tipos de saberes y con la actividad de los docentes-investigadoresextensionistas universitarios.

Compartimos la idea de Gabriela Merlisnky (2009b) de que las disciplinas científicas y técnicas resultan insuficientes para entender al ambiente como temática actual, "asimismo, han surgido nuevas formas de cooperación entre disciplinas intentando dar respuesta a las nuevas preguntas que nos reclama nuestra época" (Merlisnky, 2009:2).

Sobre esta idea señalamos que no solo es objetivo de las disciplinas científicas y técnicas entender al ambiente, sino contribuir a la elaboración de respuestas sobre la base de los saberes técnicos. En esa línea, apuntamos a que la extensión forme profesionales comprometidos con la realidad social, que se sientan interpelados sobre el sentido de la universidad y el vínculo que esta tiene y debe tener con la comunidad.

Así entendida, la extensión universitaria conlleva un proceso de enseñanza-aprendizaje en el sentido en que lo plantea Freire: "la educación verdadera es praxis, reflexión y acción del hombre sobre el mundo para transformarlo" (1964:7).

Creemos necesario que los grupos extensionistas estén constituidos por trabajadores docentes, no docentes y estudiantes de distintas disciplinas. No solo para mejorar su abordaje epistemológico a la hora de comprender y actuar en realidades complejas, sino también como práctica enriquecedora y solidaria. Consideramos que este es el único modo de poner en práctica una construcción genuinamente horizontal y respetuosa de la diversidad.

\section{Definiciones: los conflictos socioambientales}

Existe consenso en cuanto a caracterizar a los conflictos ambientales como un tipo particular de conflicto social que suele darse en el ámbito público. La noción de conflicto social representa uno de los conceptos básicos y constitutivos de las ciencias sociales, ya que resulta un aspecto inherente e ineludible de toda situación social. Los conflictos sociales son procesos, es decir, no son estáticos y tienen un desarrollo temporal. Estos procesos tienen lugar en el ámbito público, por lo que se excluyen las disputas del espacio privado. Un conflicto alude a una dinámica de oposición, controversia, disputa o protesta de actores (Santandreu y Gudynas, 1998).

Numerosos autores plantean una diferencia entre lo que se reconoce por conflicto ambiental y por conflicto socioambiental: en el primer caso, se trata de conflictos relacionados con el daño a los bienes naturales, donde la oposición proviene principalmente de actores exógenos, por lo común activistas de organizaciones ambientalistas. En el segundo caso, los conflictos también involucran a las comunidades directamente afectadas por los impactos derivados de un determinado proyecto (Walter, 2009) (Orellana, 1999). Esta distinción ha sido discutida puesto que no existe "conflicto ambiental" sin dimensión social (Fontaine, 2004), es por eso que la categoría de conflicto socioambiental reafirma que el ambiente resulta una construcción sociopolítica.

Según Folchi Donoso (2001), los conflictos ambientales no son solo aquellos que nacen de un daño ambiental sino de cualquier proceso de transformación ambiental, independientemente de su valoración. Estos conflictos tienen un mismo origen estructural: son consecuencia del sistema económico de inspiración neoliberal implementado por dictaduras militares que además coincide con el desarrollo de una "conciencia ambientalista" creciente a nivel mundial. Si bien el autor se refiere específicamente al caso chileno, también abarca al territorio latinoamericano, en donde las dictaduras y las políticas económicas neoliberales han profundizado los conflictos ambientales.

La tradición de intelectuales ambientalistas latinoamericanos señala que la expansión del capital lleva consigo un constante deterioro social y ambiental y un aumento creciente de la tasa de explotación de los recursos naturales, que se expresa en una crisis ambiental de orden mundial pero con repercusiones y ejemplos regionales y locales (Leff, 1998).

Esta crisis ambiental es inédita, ya que, en virtud de su carácter global, afecta a todas las sociedades del planeta y se ha venido gestando con intensidad creciente en los últimos 200 años. Además, en el último medio siglo se ha constatado el debilitamiento de procesos ambientales que no pueden simplemente ser sustituidos por otros. Asimismo, como expresa Merlinsky:

"esta crisis hace más visible la estrecha vinculación existente entre las relaciones que los seres humanos establecen entre sí en la producción de sus condiciones de vida, y las que como especie establecen con el conjunto del mundo natural". (2009:2)

En esta línea de pensamiento se destaca la tesis del "ecologismo de los pobres" o el "ecologismo popular", que plantea que en el 
sur existe una corriente de movilización originada en conflictos socioambientales producidos por el crecimiento económico que conlleva la extracción de recursos, expansión de vertederos y riesgos de contaminación para quienes el ambiente es la base material de sustento (Guha y Martínez Alier, 1997).

En la última década, el surgimiento de protestas en torno a la potestad de decidir sobre el uso del territorio hizo que quedara de manifiesto la dimensión política de este fenómeno. Estos "nuevos movimientos sociales" (Walter, 2009) se caracterizan por el pluralismo de valores e ideas, su orientación pragmática y la propuesta de reformas institucionales tendientes al desarrollo de sistemas que amplíen la participación en las decisiones de interés colectivo.

Esta visión, relatada y analizada por varios autores, incorpora las relaciones de poder dentro del mapa de los conflictos ambientales, ya que pone el acento en los intereses políticos y económicos, que en general tienen una misma matriz y apuntan a un modelo de desarrollo extractivo.

\section{La universidad pública y los conflictos ambientales}

Históricamente, la universidad pública fue considerada una entidad prestigiosa, independiente y capaz de opinar sobre determinados temas; un principio de autoridad fundado en que se trata de un centro de conocimiento y de investigación orientado a la formación de profesionales. Se la reconoce como una institución educativa que busca promover mejoras en la sociedad y generar materiales de divulgación.

Particularmente, el compromiso con el ambiente no ha sido una prioridad en las currículas de la educación superior en Argentina. A pesar de que numerosas universidades trabajan en proyectos de extensión o investigación con estas temáticas, los enfoques no siempre tienen una mirada compleja y transdiciplinar. Sin embargo, existen grupos que desde algunas facultades se vinculan con los movimientos socioambientales y responden a las necesidades de la comunidad a través de proyectos de acción colectiva cuyo objeto es el de contrarrestar las tendencias socialmente negativas del sistema existente y con la perspectiva de fomentar un modelo alternativo. Como ejemplo podemos citar la cátedra de Salud Socioambiental de la Facultad de Medicina de la Universidad Nacional de Rosario, las cátedras libres de Soberanía Alimentaria de la UNLP y de la Universidad de Buenos Aires, el Área de Ecología de la Universidad Nacional de General Sarmiento, el Programa Compromiso Ambiental de la Universidad Nacional del Centro de la Provincia de Buenos Aires y el Programa Ambiente y Sociedad de la Universidad Nacional del Litoral.

La ciencia moderna occidental entiende al conocimiento como un reflejo objetivo de la realidad que se construye a partir de una racionalidad metodista y sustenta la producción del conocimiento en la relación de dominio sujeto-objeto. El rol de la ciencia y la lógica de producción del conocimiento es funcional a la dinámica económica y, por lo tanto, por más que una comunidad tenga acceso ilimitado a la información, y por muy capacitados que estén sus miembros, no se va a definir la defensa de sus derechos ambientales y sociales en el debate técnico (Zegarra, 2008). Sin embargo, las ciencias ambientales nos plantean una nueva complejidad de problemáticas que precisa nuevos marcos conceptuales para su abordaje. Por un lado, el objeto de estudio y el sujeto se presentan como la misma cosa y, por otro, la infinidad de variables y la multiplicidad de niveles que explican las problemáticas trascienden la visión acotada, materialista y metodista, de las disciplinas científicas. A pesar de que se reconoce que el abordaje de los conflictos socioambientales requiere un enfoque transdisciplinar, todavía las ciencias ambientales se centran en gran medida en considerar al ambiente como objeto de estudio y no se pueden abandonar los resabios de contemplación o simplemente dejar de pensarla como disciplina (Bocero, 2007). Los conflictos socioambientales, como manifestación social y política de las problemáticas ambientales, tensionan el protocolo de abordaje que la ciencia disciplinar presupone para un problema (Merlinsky, 2007). Los mismos plantean la necesidad de estudiar los factores ambientales que derivan en una determinada situación, a la vez que es preciso abordar el problema en relación con la comunidad y con los tiempos que la circunstancia demanda. El trabajo con la comunidad y el respeto a los saberes populares generan una contradicción en el ámbito científico que todavía mantiene su esencia elitista y exclusiva, posicionándose por encima del resto de la sociedad y desde una mirada supuestamente objetiva y exenta de elementos ideológicos y políticos.

En definitiva, lo que se disputa dentro del paradigma científico es el reconocimiento de los derechos de la comunidad, no solo a un ambiente digno sino a ser considerada como sujeto capaz de comprender su situación en una relación de igualdad y reciprocidad con el resto de la sociedad.

El proceso histórico de lucha de las comunidades relegadas y puestas en inferioridad, consecuencia del etnocentrismo de los países occidentales, se expresa en la ciencia que sostiene su nicho de ideas fundantes en estas sociedades llamadas centrales (Walsh, 2007). El conocimiento occidental, autoproclamado como universal y superior, constituye la racionalidad discriminante del resto de las sociedades periféricas, consideradas subalternas.

En ese marco, "lo ambiental" representa uno de los grandes desafíos que enfrenta la universidad en el siglo XXI y los conflictos socioambientales se configuran, entonces, como un campo de estudio amplio, donde convergen distintas miradas e intereses; es un punto de articulación interdisciplinario, objeto de preocupación y estudio para diferentes áreas de las ciencias (Merlinsky, 2007). Es además objeto de preocupación de distintos ámbitos, entre ellos 


\section{6}

lo que se disputa dentro del paradigma científico es el reconocimiento de los derechos de la comunidad, no solo a un ambiente digno sino a ser considerada como sujeto capaz de comprender su situación en una relación de igualdad y reciprocidad con el resto de la sociedad

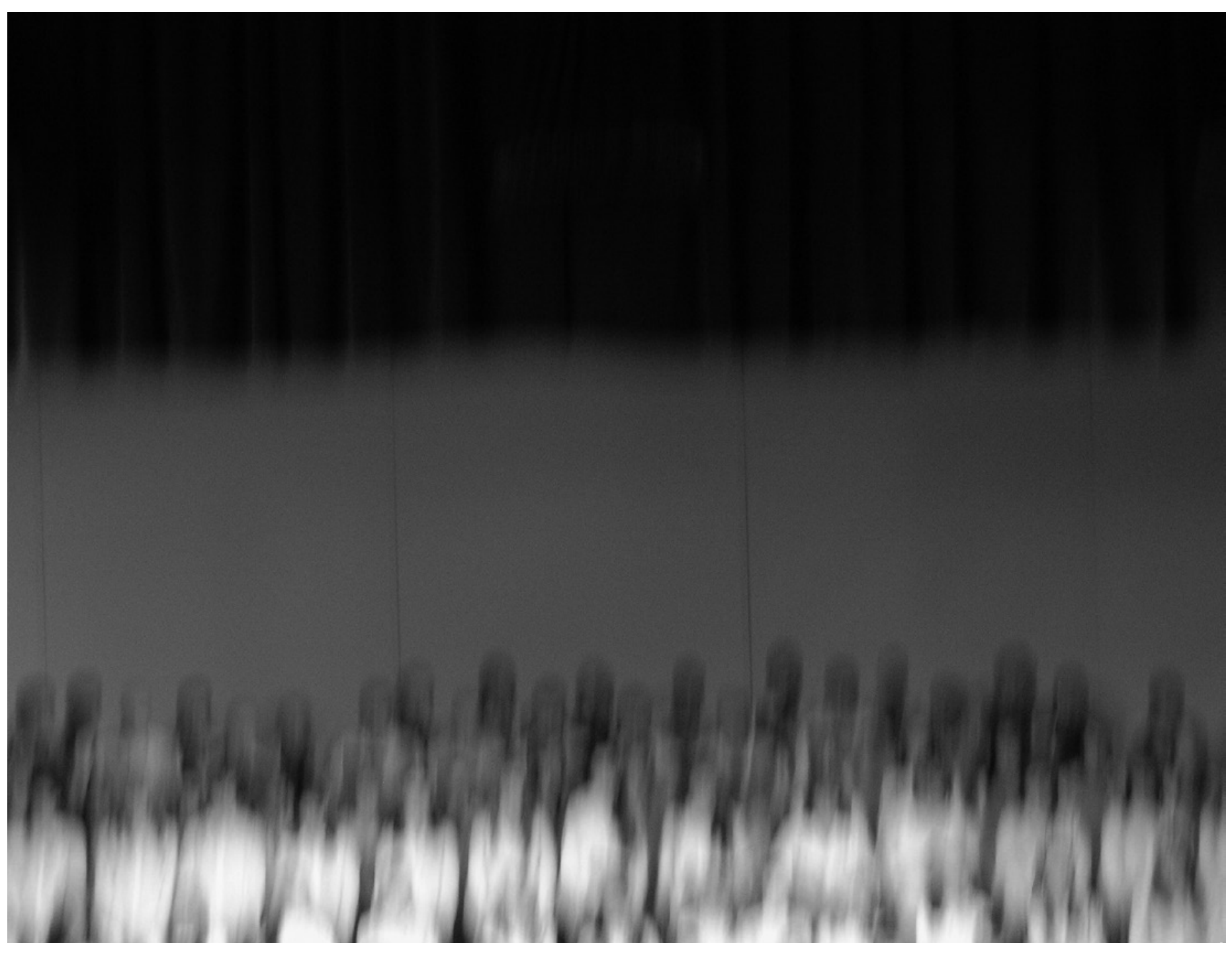


las empresas y los gobiernos, los vecinos, las ONG, las asambleas ambientales, los medios de comunicación, además de la comunidad científica y universitaria.

Se plantea así, en el ámbito académico, un nuevo paradigma descrito por Merlinsky:

"lo ambiental no puede reducirse a un problema demográfico, tecnológico, o meramente económico, para dar paso a una visión de creciente complejidad, que nos demanda nuevas formas de colaboración e interacción entre las ciencias humanas y las naturales". (2009:2)

\section{Los desafíos regionales del PAEU}

En nuestra experiencia particular como programa ambiental, hemos ido abordando en conjunto con la comunidad diversos ejes de trabajo que se asocian a problemáticas de la región. Paralelamente, organizaciones, profesionales y grupos ambientalistas de la zona vienen elaborando una Agenda Ambiental con las problemáticas más urgentes a resolver en la región y sostienen en dicho documento que la contaminación industrial, la contaminación electromagnética y la gestión de los residuos son algunos de los temas más preocupantes para el Gran La Plata. En este sentido, la Universidad juega un rol fundamental en la validación de las premisas que surgen de los reclamos de los pobladores. Desde el PAEU propusimos los siguientes ejes de trabajo como forma de organización de los proyectos y líneas de intervención:

\section{Monitoreo Ambiental: en el Programa se desarrollan} monitoreos de agua, aire y de condiciones laborales. Si bien el agua es un elemento imprescindible para el desarrollo de la vida y su acceso es un derecho esencial para el ser humano, existen numerosas zonas de nuestro país en donde esto se ve vulnerado. En especial, en nuestra región, en la zona de humedales periurbanos, y en la desembocadura del Arroyo del Gato sobre el Río de La Plata, se realizan monitoreos de la calidad del agua. Esta zona es ambientalmente crítica ya que en la misma se encuentran el Puerto La Plata, la toma de agua potable y desagües cloacales e industriales.

Por otra parte, se realiza un monitoreo de condiciones laborales en pobladores cercanos a las industrias, dado que nuestra región cuenta con un importante desarrollo industrial y productivo que afecta no solo al ambiente de los trabajadores sino de todos los pobladores que habitan las cercanías de estos emprendimientos. En este sentido, se reconoce como principal actividad industrial la del Polo Petroquímico de Berisso y Ensenada, uno de los más grandes de Argentina. Frente a esta problemática el Programa viene articulando con trabajadores de la Cooperativa Futuro Ensenadense, de la Unión Vecinal Mosconi, quienes poseen una larga trayectoria de lucha por un barrio más saludable.
En este marco se han efectuado diferentes estudios para determinar los efectos que ocasiona la contaminación en la salud, específicamente en la función respiratoria y en la salud ocular. Los resultados mostraron que la población presenta un desbalance nutricional, hábitos tóxicos (con predominio del hábito tabáquico), alteraciones en los valores de colesterol y triglicéridos, alteraciones en los valores tanto de eritrocitos como de leucocitos, afecciones respiratorias (del tipo irritativas y alérgicas) y afecciones oculares como irritaciones y ojo seco. Esto nos motorizó a trabajar en torno a la prevención y la promoción de la salud, tanto con la Cooperativa como con la Escuela Técnica $N^{\circ} 2$ de Ensenada también lindante con el Polo Petroquímico-, en donde llevamos tres años ininterrumpidos de talleres.

En un inicio, nos basamos en las problemáticas encontradas y luego los participantes fueron proponiendo las temáticas para los talleres de su interés, ya sea por situaciones personales o por las de su grupo familiar. Ambas instituciones, la Escuela $N^{\circ} 2$ y la Cooperativa, son claves en el cuidado socioambiental del barrio Mosconi de Ensenada. La escuela, además, tiene una tecnicatura en Química y capacidad de monitorear algunas condiciones ambientales del barrio, a la vez que puede desarrollar y proponer mejoras. Del mismo modo, la Cooperativa es un actor fundamental para la realización de cambios y reformas, e históricamente trabaja por mejorar las condiciones ambientales y de habitabilidad del barrio.

2. Tecnología ambiental: la tecnología es un aspecto inherente y, de algún modo, identitario de la humanidad. Los procesos sociales siempre estuvieron signados por modos tecnológicos de suplir necesidades desde el surgimiento de la especie, el nomadismo, la revolución neolítica y muy posteriormente la revolución industrial. La hegemonía occidental del conocimiento y de la producción desencadenaron en los últimos 500 años formas de producción y aplicación tecnológica peligrosamente destructivas en nuestra región. En este marco, las tecnologías desempeñan un papel central en los procesos de cambio social, demarcan posiciones y conductas de los actores, condicionan estructuras de distribución social, costos de producción, acceso a bienes y servicios, generan problemas sociales y ambientales y facilitan o dificultan su resolución. Desde el Programa se propone una línea de desarrollo tecnológico con dos ejes: por un lado, tecnologías y estratégicas de recuperación de ambientes acuáticos contaminados y, por otro lado, tecnologías que garanticen el al acceso seguro al agua potable, teniendo en cuenta que la mayor dificultad para lograrlo es consecuencia de los actuales modos de producción y actividad industrial que la contaminan.

En función de este primer objetivo, el PAEU está llevando a cabo un proceso tecnológico integral de recuperación de lagunas pampeanas que están afectadas por el enriquecimiento de 
nutrientes, o eutrofización, producto de las actividades agropecuarias y el desarrollo urbano. En este marco, se construyó una planta piloto en el Zoológico de la Plata con el objetivo adicional de exponerla públicamente y, de manera explicativa, visualizar una experiencia alternativa de desarrollo tecnológico focalizada en la recuperación de uno de los cuerpos de agua dulce característicos del humedal pampeano. Esto fue posible de construir por la colaboración de Faisán, una Pyme argentina dedicada a dar servicio tecnológico en tratamiento de aguas ubicada en Florencio Varela, provincia de Buenos Aires. A tal efecto se firmó un convenio con la empresa y con el Zoológico de La Plata.

Con relación al segundo objetivo, se trabaja para la eliminación de nitratos de aguas subterráneas a través de la preparación y caracterización de arcillas aniónicas, del tipo hidrotalcita, para ser empleadas como absorbentes de nitratos en aguas de consumo humano. En las zonas periféricas del partido de La Plata y alrededores no poseen acceso a la red de agua potable y se ha determinado, a través de monitoreos, que los niveles de nitratos presentes en el agua exceden a los permitidos.

En el mismo eje, se desarrollan sistemas de potabilización de aguas superficiales a pequeña escala a fin de garantizar un acceso seguro a familias, productores o instituciones que no tienen acceso a la red. Se implementan y adaptan tecnologías tradicionales ampliamente utilizadas, de modo de refuncionalizar materiales y equipos económicos comercialmente disponibles. En este marco, se está trabajando con tres instituciones educativas isleñas del municipio de Zárate y un productor de Ensenada, en la costa del Río de La Plata, con los cuales se elaboraron propuestas y asesoramientos para su implementación.

La soberanía tecnológica es una de las necesidades más estructurales para pretender alcanzar la soberanía económica y política de nuestro país pero, si implica reproducir el modelo de desarrollo extractivista, se atenta contra nuestras necesidades reales.

3. Agrotóxicos: en las últimas décadas se ha intensificado el uso de agrotóxicos en la agricultura, por lo cual es una fuente importante de contaminantes que ingresan al ambiente.

En ese sentido, en el PAEU funciona activamente el Espacio Multidisciplinario de Interacción Socioambiental (EMISA), un proyecto de extensión que cuenta con más de 30 integrantes de distintas áreas como la ingeniería, las ciencias exactas, naturales, médicas, humanidades y ciencias la educación, que tiene por objetivo aplicar herramientas de la química ambiental como un aporte a la resolución de conflictos sociales derivados del uso de agrotóxicos.

De esta manera, y a través de la demanda social, se contribuye al diagnóstico ambiental con una herramienta más en pos de la resolución del conflicto socioambiental en cuestión. A medida que se fue consolidando, se organizó en distintas líneas de trabajo, tales como plaguicidas en alimentos, escuelas fumigadas, campamentos sanitarios, ventana de articulación social y un área de comunicación. Se trabaja con el Movimiento Campesino de Santiago del Estero (MOCASE - Vía Campesina), con productores de la Isla Paulino (Berisso, provincia de Buenos Aires) y con el Instituto de Investigación y Desarrollo para la Agricultura Familiar (IPAF), dependiente del Instituto Nacional de Tecnología Agropecuaria (INTA).

4. Educación ambiental: es un proceso que nos permite comprender a las sociedades humanas y como individuos en interdependencia con nuestro entorno.

Desde el Programa se trabaja en esta línea con una escuela secundaria y con estudiantes y graduados de diferentes facultades de la UNLP. A comienzo de 2013 se realizó un convenio con la Escuela Técnica $N^{\circ} 2$ de Ensenada para que los alumnos del $6^{\circ}$ año de la Tecnicatura en Química pudieran hacer las prácticas profesionalizantes necesarias para graduarse en las instalaciones del PAEU.

Ese convenio asegura la formación en cuanto a conocimientos técnicos sobre la correcta toma de muestras de agua en cuerpos superficiales, el almacenamiento de las mismas y el análisis fisicoquímico y microbiológico, lo cual les permitió monitorear los arroyos aledaños a su escuela.

Por otra parte, implica que los alumnos se trasladen a la Facultad y que este sea el primer paso en una institución universitaria, de manera de conocer sus dinámicas y profesionales. A su vez, con los resultados obtenidos, los alumnos pudieron graduarse y también presentarlos en la Feria Provincial de Ciencias y Tecnología.

En cuanto a la docencia puertas adentro de la Universidad, se ha puesto nuestro laboratorio al servicio de tesinas y trabajos para la Licenciatura en Química y Tecnología Ambiental y para otras carreras de nuestra Facultad. A la fecha tenemos una tesina finalizada y dos en vías de desarrollo. De la misma forma, se han realizado tesis doctorales, una finalizada y tres en desarrollo.

5. Espacio de debate: en la práctica de cada día nos encontramos con la necesidad de debatir colectivamente. El conocimiento científico en un proceso de creación constante que se logra mediante el trabajo constructivo, en relación continua con otros o con los procesos de la realidad, donde conocer implica interpretar y usar el error como punto de emergencia de nuevos conocimientos. Dentro del PAEU fomentamos las discusiones teóricas respecto de los temas ambientales y generamos espacios de intercambio y debate. En ese sentido, realizamos el curso de posgrado 
"Elementos de Ecología Política: Introducción a una práctica multidisciplinaria en áreas suburbanas", que tuvo lugar del 1 al 5 de agosto de 2015 en la Facultad de Ciencias Exactas de la UNLP. El equipo docente estuvo encabezado por el Dr. Héctor Alimonda, del Instituto de Investigaciones "Gino Germani" de la Facultad de Ciencias Sociales, UBA, y del CPDA de la Universidade Federal Rural de Rio de Janeiro. Alimonda disertó sobre la Ecología Política, sus desafíos y perspectivas, la relación histórica entre sociedades y naturalezas y el papel del poder social. Además, problematizó la dimensión latinoamericana de la Ecología Política antes y después de la conquista.

También hicieron sus aportes la Mg. Melina Tobías y las Dras. Soledad Fernández Bouzo y Mariana Schmidt, del Grupo de Estudios Ambientales del Área de Estudios Urbanos del Instituto de Investigaciones "Gino Germani". Las investigadoras aportaron herramientas conceptuales para pensar los conflictos ambientales a partir de estudios de casos.

Con sus exposiciones problematizaron el territorio como escenario de manifestación y como espacio "valorizado" por el conflicto y dieron algunas pistas para la construcción de una sociología de los conflictos ambientales.

El curso incluyó un recorrido por el Gran La Plata y sus problemáticas ambientales, en el cual se reconoció el territorio, su flora y fauna y los conflictos socioambientales en torno a la contaminación industrial, la gestión de residuos, el ordenamiento territorial y los desmontes. Finalizó con las exposiciones del Dr. Darío Andrinolo y la Lic. Florencia Yanniello, ambos del Área de Toxicología del Centro de Investigaciones del Medio Ambiente y del PAEU, quienes realizaron una reconstrucción de la historia ambiental de la región y aportaron algunos ejes sobre cómo abordar el análisis y la divulgación de los conflictos ambientales en el marco de los debates de comunicación, ciencia y sociedad.

6. Comunicación: el acceso a la información sobre la calidad ambiental de nuestra región es complejo y limitado. No solo porque la mayoría de los estudios pedidos por entes oficiales o privados goza de cláusulas que impiden la libre divulgación de los datos, sino porque no existen mecanismos de tratamiento y divulgación que informen con el objetivo de entregar herramientas analíticas que posibiliten el empoderamiento por parte de los ocupantes y usuarios de esos espacios.

La información y la comunicación dominantes son parte del paradigma individualista neoliberal que debe ser contrastado con un pensamiento que considere "nuestro" territorio, que nos aúna e iguala en tanto todos somos sus pobladores.

La participación dentro del Programa de una profesional de la comunicación en temas ambientales permitió construir y ahondar en paradigmas alternativos de convivencia en nuestro ambiente. Por esto, nos propusimos el desarrollo de un sitio web que comunique todas las líneas de acción del PAEU y muestre los resultados obtenidos y proyectos generados. En este sentido, tenemos una página (Portal A: www.portala.exactas.unlp.edu. ar) y un perfil de Facebook (Programa Ambiental de Extensión Universitaria) en donde se pueden ver las acciones del PAEU. Actualmente estamos en proceso de generar una plataforma tipo SIG (Sistema de Información Geográfica), de libre acceso, que tendrá la información de los diferentes monitoreos.

Por otra parte, el Programa ha intervenido frente a la demanda de la población en problemáticas socioambientales como la del electromagnetismo en Berazategui, en el conurbano bonaerense sur. Es por ello que se evaluó el impacto de la subestación eléctrica Rigolleau en la salud humana. Desde el PAEU se convocó a especialistas físicos y toxicólogos, quienes analizaron los documentos aportados por los vecinos y durante los años 2012 y 2013 se desarrolló un estudio epidemiológico en donde se demostró el daño de las ondas electromagnéticas en la salud de la población. Este material público, generado en la Universidad, permitió a las poblaciones afectadas poder accionar legalmente frente a las instituciones correspondientes.

Otro caso interesante, fue el del Análisis de Coliformes Fecales en el Arroyo del Gato en las cercanías de la planta depuradora de efluentes cloacales de la empresa Aguas Bonaerenses SA (ABSA) en la localidad bonaerense de Ringuelet. El mismo fue solicitado por la Clínica Jurídica de Derecho del Consumidor, de la Facultad de Ciencias Jurídicas y Sociales de la UNLP. El informe forma parte de una presentación ante la justicia de la Clínica Jurídica debido al incumplimiento por parte de ABSA del tratamiento de desechos cloacales antes de ser volcados al arroyo del Gato.

De esta forma, mediante la elaboración de informes de carácter público y su difusión en Portal A y nuestras redes sociales, pretendemos contribuir a la divulgación de las temáticas ambientales de manera abierta, pensando en la utilidad de estos materiales para mejorar la situación sociosanitaria de la comunidad.

\section{Conclusiones}

Desde el punto de vista institucional, la generación de estructuras académicas nuevas como los programas extensionistas, deberían potenciar las practicas integrales, dando lugar a nuevos espacios en donde la docencia y la investigación puedan despegarse de la objetividad y promover activamente transformaciones en alianza con distintos sectores de la población.

A partir de la creación de nuestro programa, se acompañó a una gran cantidad de actores sociales en sus demandas, participando en acciones comunes, destinadas a transformar las propias circunstancias. Tal es así que coordinamos con la Escuela Técnica 
$N^{\circ} 2$ de Ensenada, con la Cooperativa Futuro Ensenadense, con el Foro por la Salud y el Ambiente de Berazategui, las Clínicas Jurídicas de la Facultad de Ciencias Jurídicas y Sociales, distintos centros vecinales, municipios, organizaciones barriales y ONG.

Para profundizar en esta tarea, vemos preciso mejorar la calidad de nuestras intervenciones e investigaciones extensionista, modificar las formas de gestión de los recursos, enriquecer la comunicación pública de las experiencias y resultados, profundizar en discusiones de forma colectiva, trabajar al unísono desde los distintos programas y proyectos, y fundamentalmente, formar parte activa dentro de cada comunidad.

Hemos notado que los principales conflictos ambientales, muchas veces transformados en candentes temas políticos, están subrepresentados en las líneas de trabajo de la Universidad. La contaminación ambiental, la degradación de los espacios naturales, el uso indiscriminado de agroquímicos, el derecho a la información y el derecho a la toma de decisiones ambientales por parte de la comunidad, son algunos de los temas que la universidad pública debe abordar desde sus tres pilares. La extensión ambiental es, en este sentido, una posibilidad de incorporar otros temas y actores hoy ausentes o marginados.

En esa línea, una de las mayores contribuciones de la extensión es, entonces, llevar al seno de la universidad las contradicciones que presenta nuestro actual modelo de desarrollo neoliberal, extractivista y basado en los derechos individuales, para contribuir a sociedades más justas y sustentables. De esta forma, la extensión, los proyectos, y programas como formas superadoras, son los espacios necesarios para legitimar nuevas formas de producir conocimiento en pos de resolver problemáticas sociales y ambientales.

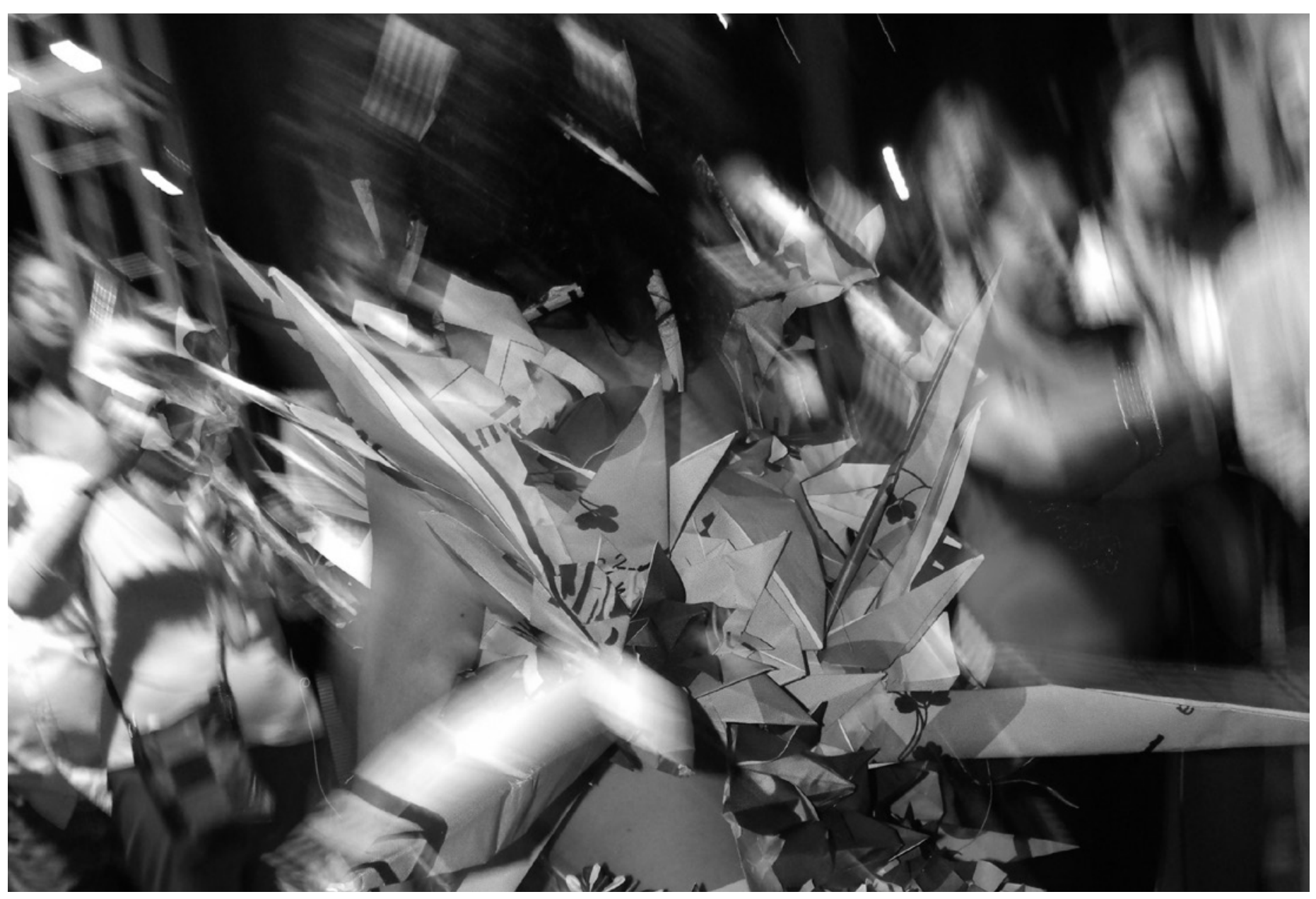

(c) Cecilia lucci 
Hemos notado que los principales conflictos ambientales, muchas veces transformados en candentes temas políticos, están subrepresentados en las líneas de trabajo de la Universidad

\section{Referencias bibliográficas}

Argumedo, M. A. y otros (2002). Las estrategias de formación de sujetos en los proyectos de extensión universitaria en la UNLP. En Prati, M. (Ed.), La Universidad Cautiva. Legados, Marcas y Horizontes (pp. 295-309). La Plata: Ediciones Al Margen.

Bocero, S. y Natenzon, C. (2007). La dimensión ambiental del territorio en América Latina: aportes para su discusión. En Fernández Caso, M. V. y Gurevich, R. (Ed.), Geografía. Nuevos temas, nuevas preguntas. Un temario para su enseñanza (pp. 65-94). Buenos Aires: Biblos.

Caldelari, M. (2002). Apuntes sobre extensión universitaria en la UBA, 1955-1966. En Prati, M. (Ed.), La Universidad Cautiva. Legados, Marcas y Horizontes (pp. 43-56). La Plata: Ediciones Al Margen.

Consejo Interuniversitario Nacional (CIN) (1997). Inclusión de la función extensión en el presupuesto 1998 para el sistema universitario. Recuperado de: http://www. cin.edu.ar/result.php? $\mathrm{p}=10 \&$ offset $=25 \& 1=1$ \&palabras=extensi $\% F 3 n \#$ (consultado el 09/12/1997).

Estatuto Universidad Nacional de La Plata (1996). Título VIII. Capítulo I: De la Extensión Universitaria. Art. 106. Recuperado de: http://www.unlp.edu.ar/ uploads/docs/estatuto_universitario_con_diseno.pdf

— (2008). Título II: Funciones. Capítulo III: de la Extensión. Art. 17². Recuperado de: http://www.unlp.edu.ar/uploads/docs/estatuto_2008.pdf

Folchi Donoso, M. (2001). Conflictos de contenido ambiental y ecologismo de los pobres: no siempre pobres, ni siempre ecologistas. Ecología política, (22), 79-101. Fontaine, G. (2004). Enfoques Conceptuales y metodológicos para una sociología de los conflictos ambientales. En Cardenas M. y Rodríguez M. (Ed.), Guerra, Sociedad y Medio Ambiente (pp. 503-533). Bogotá: Foro Nacional Ambiental. Freire, P. (1964). La educación como práctica de libertad. México: Siglo XXI Editores. González González, G. R. y González Fernández-Larrea M. (2003). Extensión universitaria: principales tendencias en su evolución y desarrollo. Revista Cubana de Educación Superior, (1), 15-26.
Guha R. y Martínez Alier J. (1997). Varieties of environmentalism. Essays North and South. London: Earthscan.

Leff, E. (1998). Saber Ambiental: Sustentabilidad, racionalidad, complejidad, poder. México: Siglo XXI Editores.

Martínez Alier, J. (1995). De la Economía ecológica al ecologismo popular. Montevideo: Nordan Comunidad - ICARIA.

Merlinsky, M. G. (2007). Conflicto ambiental, organizaciones y territorio en el Área Metropolitana de Buenos Aires. En Sociedad Civil y Desarrollo Local (pp. 585 605). México: Porrúa.

(2009): Conflictos ambientales y territorio (clase), en el curso "Ecología política en el capitalismo contemporáneo". Programa Latinoamericano de Educación a Distancia, Centro Cultural de la Cooperación Floreal Gorini. Buenos Aires.

Merlinsky, M. G.; Herzer, H. M., y Prévôt Schapira, M. (2009). Atravesando el río: la construcción social y política de la cuestión ambiental en Argentina: dos estudios de caso en torno al conflicto por las plantas de celulosa en el Río Uruguay y al conflicto por el saneamiento de la Cuenca Matanza-Riachuelo. Tesis doctoral. Universidad de Buenos Aires - Universidad París VIII.

Orellana, R. (1999). Conflictos... ¿sociales, ambientales, socioambientales?... Conflictos y controversias en la definición de los conceptos. En Ortiz, P. (Ed.), Comunidades y Conflictos Socioambientales: experiencias y desafiaos en América Latina (pp. 331-344). Quito: Abya-Yala.

Santandreu, A. y Gudynas, E. (1998). Ciudadanía en movimiento. Participación ciudadana y conflictos ambientales. Montevideo: CLAES, FESUR y Trilce.

Walsh, C. (2007). ¿Son posibles unas ciencias sociales/culturales otras? Reflexiones en torno a las epistemologías decoloniales. Nómadas, (26), 102-113.

Walter, M. (2009). Conflictos ambientales, socioambientales, ecológicos distributivos, de contenido ambiental... Reflexiones sobre enfoques y definiciones. Boletín de Centro de Investigación para la Paz, (6), 1-9. 